\title{
LACTANCIA MATERNA COMO FACTOR PROTECTOR PARA ENFERMEDADES PREVALENTES EN NIÑOS HASTA DE 5 AÑOS DE EDAD EN ALGUNAS INSTITUCIONES EDUCATIVAS DE COLOMBIA 2009. ESTUDIO DE CORTE TRANSVERSAL
}

\author{
A cross-sectionall study of maternal breastfeeding as \\ protection factor for prevalent diseases in children \\ up to 5 years of age in some Colombian educational \\ institutions, 2009 \\ María Camila Alzate-Meza*, César Arango, MD**,JoséJaime Castaño-Castrillón, Fis. MSc***, \\ Angélica María Henao-Hurtado*, María Margarita Lozano-Acosta*, \\ Geovanny Muñoz-Salazar*, Natalia Andrea Ocampo-Muñoz*, \\ Sarah Violeta Rengifo-Calderón*, Lina Marcela Tovar-Orozco*, \\ Saúl Hernando Vallejo-Chávez*
}

Recibido: agosto 19/10 - Aceptado: febrero 16/11

\section{RESUMEN}

Objetivo: establecer la frecuencia de empleo y explorar el papel que tiene la lactancia materna exclusiva hasta los 6 meses como factor protector para enfermedades prevalentes, en niños hasta de 5 años de edad en una determinada población colombiana.

Materiales y métodos: se realizó un estudio de corte transversal en algunas instituciones educativas a nivel de jardín infantil de los municipios de Palestina, Ibagué y Pereira (Colombia). La escogencia de la población se efectuó mediante un muestreo

* Estudiante X semestre, Facultad de Medicina, Universidad de Manizales. Manizales (Colombia)

** Pediatra, docente Pediatría, Facultad de Medicina, Universidad de Manizales, Hospital Infantil Universitario de Manizales. Manizales (Colombia).

*** Profesor Titular, Director Centro de Investigaciones, Facultad de Medicina, Universidad de Manizales. Manizales (Colombia). Correo: jcast@ umanizales.edu.co. por conveniencia. Se evaluaron las características basales, tipo de alimentación, tiempo de lactancia materna, tiempo de alimentación complementaria, enfermedades respiratorias, apendicitis, enfermedad diarreica aguda, diabetes mellitus, dermatitis, reflujo gastroesofágico, desnutrición, obesidad y afección cardiorrespiratoria.

Resultados: participaron en el estudio 311 niños. Se encontró una frecuencia de lactancia materna del 92\% (98,1\% Ibagué, 90,5\% Pereira, $87,9 \%$ Palestina) y una asociación significativa entre la lactancia materna y bronquiolitis, reflujo gastroesofágico, síndrome bronco obstructivo, desnutrición y afección cardior respiratoria.

Conclusiones: se confirma la importancia de la lactancia materna como factor protector para enfermedades prevalentes en la infancia.

Palabras clave: lactancia materna, control de riesgo, prevención primaria, pediatría. 


\section{SUMMARY}

Objective: establishing the frequency of use and exploring the role of exclusive maternal breastfeeding for up to six months as a protection factor against prevalent diseases in children up to 5 years of age in a determined Colombian population.

Materials and methods: a cross-sectional study was carried out in some kindergartens in the towns of Palestina, Ibagué and Pereira (Colombia). The study population was chosen by convenience sampling. Base characteristics such as type of feeding, maternal breastfeeding time, complementary feeding time, respiratory disease, appendicitis, acute diarrheic disease, diabetes mellitus, dermatitis, gastroesophageal reflux, malnutrition, obesity and cardiorespiratory affection were evaluated.

Results: 311 children participated in the study. 92\% maternal breastfeeding frequency was found $(98.1 \%$ in Ibagué, $90.5 \%$ Pereira, $87.9 \%$ in Palestina) and a significant association was found between maternal breastfeeding and bronchiolitis, gastroesophageal reflux, broncho-obstructive syndrome, malnutrition and cardiorespiratory affection.

Conclusions: the importance of maternal breastfeeding was confirmed as a protection factor for prevalent diseases during infancy.

Key words: maternal breastfeeding, risk control, primary prevention, pediatrics.

\section{INTRODUCCIÓN}

La leche materna constituye, por si sola, el mejor alimento y la mejor bebida al contener los minerales y nutrientes necesarios que deben darse en forma exclusiva a un niño o una niña hasta los seis meses de vida, acompañada de otros alimentos hasta los dos años. ${ }^{1}$ Los beneficios de la lactancia materna sobre la salud infantil son amplios, destacándose la participación importante que este tipo de alimentación ha tenido en la disminución de la mortalidad infantil, y algunas enfermedades prevalentes en la infancia. ${ }^{1}$ Se afirma que anualmente la prolongación del período de lactancia del seno materno, podría salvar la vida a 1 millón de niños menores de 1 año en los países en desarrollo. ${ }^{2}$ La leche materna contribuye a la disminución de la prevalencia e incidencia de desnutrición. ${ }^{3}$ La lactancia materna es la medida más efectiva y menos costosa para evitar desnutrición durante los primeros meses de vida, ${ }^{4}$ también reduce el riesgo de diabetes mellitus insulino-dependiente, ${ }^{5}$ de alergias, ${ }^{5}$ diarreas e infección respiratoria. ${ }^{6,7}$

A pesar de todos estos beneficios, en los últimos 60 años se ha observado un descenso progresivo en la práctica de lactancia natural en el mundo. Este fenómeno ha tenido múltiples consecuencias sobre la población. Para los países en vías de desarrollo, el abandono precoz de la lactancia materna ha significado efectos a corto plazo sobre la salud del niño menor, aumentando el riesgo de muerte, morbilidad, desnutrición y la frecuencia de hospitalizaciones y el elevado costo económico que representa su reemplazo por leches artificiales. ${ }^{8} \mathrm{La}$ UNICEF revela que un 95\% de niños y niñas son amamantados cuando nacen, pero en Colombia esta proporción baja al 61\%. ${ }^{9}$

Existe controversia sobre los beneficios de la lactancia materna hasta el primer año de vida, ya que no se ha observado una asociación entre lactancia materna y la prevención de las infecciones respiratorias durante este período de tiempo de forma global, puesto que sólo en el segundo trimestre de vida, hay una asociación protectora frente a las infecciones respiratorias. ${ }^{10}$

En vista de lo anterior, la presente investigación tiene como objetivo establecer la frecuencia de la lactancia materna exclusiva y su asociación, en cuanto a la prevención de enfermedades, en niños de 1 a 5 años, en una población colombiana determinada.

\section{MATERIALES Y MÉTODOS}

Se realizó un estudio de corte transversal. La población estuvo conformada por niños entre 1 y 5 
años de edad, de 4 instituciones educativas a nivel de Jardín Infantil, 2 en el municipio de Palestina (con 140 niños), 1 en la ciudad de Ibagué (con 106 niños) y 1 en la ciudad de Pereira ( con 63 niños). Estas ciudades están situadas en la región andina central en Colombia (Suramérica). La escogencia de la población se efectuó mediante un muestreo por conveniencia. El estudio fue aprobado por el comité de ética e investigación de la Facultad de Medicina de la Universidad de Manizales.

Se elaboró un instrumento de 11 preguntas, para ser respondido por las madres de los niños participantes, con el apoyo de los investigadores. Previa a la aplicación del instrumento, se explicó a cada una de las madres en qué consistía el trabajo, se aclararon dudas respecto a éste, y se llenó un formulario de consentimiento informado. Se evaluaron las variables demográficas, las patologías susceptibles a la presencia de lactancia materna, y los detalles de la lactancia materna. Para probar el instrumento desarrollado, el 21 de agosto de 2009 se realizó una prueba piloto con 11 madres en el municipio de Palestina. La aplicación definitiva del instrumento se efectuó en septiembre del mismo año. Se recolectó la información durante aproximadamente dos semanas en las diferentes ciudades.

Se recolectaron las siguientes variables en la población de estudio: edad (años), sexo (femenino, masculino), ciudad, procedencia (urbana y rural), estrato social (del 1 al 6), tipo de familia (familia nuclear, familia extensa o consanguínea, familia monoparental, familia de madre soltera, familia de padres separados), número de hermanos, edad materna, tipo de alimentación (leche materna, alimentación complementaria o ambas), alimentación complementaria simultánea con lactancia materna, lactancia materna, tiempo de lactancia materna, tiempo de alimentación complementaria; la presencia de neumonía, asma, bronquiolitis, rinofaringitis, faringoamigdalitis, síndrome broncoobstructivo, apendicitis, enfermedad diarreica aguda, diabetes mellitus, dermatitis de contacto, dermatitis atópica, rinitis, sinusitis, reflujo gastroesofágico, desnutrición, obesidad, afección cardiorrespiratoria, otra y patología actual.

Para el análisis de los datos, las variables nominales se describieron mediante proporciones y su respectivo intervalo de confianza, las variables numéricas continuas a través de medidas de tendencia central (promedio) y dispersión (desviación estándar). La relación entre variables nominales, se probó utilizando la prueba de $\chi^{2}$ y entre variables nominales y continuas mediante pruebas $\mathbf{t} \mathrm{o}$ análisis de varianza, según el caso. Todos los análisis se realizaron con una significancia $\alpha=0,05$. Las bases de datos se construyeron empleando el programa Excel $^{\circledR}$ (Microsoft Corporation) y los análisis estadísticos se efectuaron mediante el programa PASW 18 (SPSS ${ }^{\circledR}$ Inc.).

\section{RESULTADOS}

Se obtuvo información de 311 participantes. La caracterización demográfica de esta población se encuentra en la tabla 1. El promedio de edad de los niños fue de 4.37 años (lc 95\%:4,24-4,49), el promedio de la edad de las madres en el momento del embarazo fue 23,93 años (IC 95\%:23,22-24,64), 286 tuvieron lactancia materna y 25 no, el promedio de tiempo de lactancia materna fue de 10,64 meses (IC 95\%:9,6-11,7) y en promedio, se inició la alimentación complementaria a los 4,76 meses (IC 85\%:4,42-5,1).

Al comparar al grupo que tuvo lactancia materna con aquel que no recibió lactancia, se encontraron diferencias significativas con relación a la ciudad donde viven $(p=0,013)$ y el tipo de familia $(p=0,024)$. Se observó que la mayor proporción de lactancia se encuentra en familias nucleares, 95\%, y monoparentales, $100 \%$.

En lo referente a las patologías referidas por las madres, la más frecuente fue enfermedad diarreica aguda (37,1\%, IC 95\%:31,5\%-42,9\%), seguida de rinofaringitis (27,4\%, IC 95\%: 22,6\%-32,8\%), faringoamigdalitis (19\%, IC 95\%:14,9\%-23,9\%), 


\begin{tabular}{|c|c|c|c|c|c|c|}
\hline & & \multicolumn{4}{|c|}{ ¿Tuvo lactancia materna? } & \multirow[b]{3}{*}{ p } \\
\hline & & \multicolumn{2}{|c|}{ No } & \multicolumn{2}{|c|}{ Si } & \\
\hline & & $\mathbf{N}$ & $\%$ & $\mathbf{N}$ & $\%$ & \\
\hline \multirow{2}{*}{ Sexo } & $\mathrm{F}$ & 16 & $10,3 \%$ & 139 & $89,7 \%$ & \multirow{2}{*}{0,140} \\
\hline & M & 9 & $5,8 \%$ & 147 & $94,2 \%$ & \\
\hline \multirow{3}{*}{ Ciudad } & Ibagué & 2 & $1,9 \%$ & 104 & $98,1 \%$ & \multirow{3}{*}{0,013} \\
\hline & Palestina & 17 & $12,1 \%$ & 123 & $87,9 \%$ & \\
\hline & Pereira & 6 & $9,5 \%$ & 57 & $90,5 \%$ & \\
\hline \multirow{4}{*}{ Estrato } & 1 & 9 & $8,3 \%$ & 99 & $91,7 \%$ & \multirow{4}{*}{0,111} \\
\hline & 2 & 11 & $6,5 \%$ & 159 & $93,5 \%$ & \\
\hline & 3 & 5 & $20,0 \%$ & 20 & $80,0 \%$ & \\
\hline & 4 & 0 & $0 \%$ & 7 & $100,0 \%$ & \\
\hline \multirow{2}{*}{ Procedencia } & Rural & 4 & $9,1 \%$ & 40 & $90,9 \%$ & \multirow{2}{*}{0,739} \\
\hline & Urbano & 20 & $7,6 \%$ & 242 & $92,4 \%$ & \\
\hline \multirow{6}{*}{ Familia } & Cons/ext & 0 & $0 \%$ & 18 & $100,0 \%$ & \multirow{6}{*}{0,024} \\
\hline & Fam/mon & 0 & $0 \%$ & 3 & $100,0 \%$ & \\
\hline & $\mathrm{Mad} / \mathrm{sol}$ & 5 & $10,9 \%$ & 41 & $89,1 \%$ & \\
\hline & Mono & 1 & $12,5 \%$ & 7 & $87,5 \%$ & \\
\hline & Nuclear & 9 & $4,9 \%$ & 173 & $95,1 \%$ & \\
\hline & $\mathrm{Pad} / \mathrm{sep}$ & 10 & $18,5 \%$ & 44 & $81,5 \%$ & \\
\hline \multirow{5}{*}{ Número hermanos } & 0 & 8 & $10,1 \%$ & 71 & $89,9 \%$ & \multirow{5}{*}{0,113} \\
\hline & 1 & 7 & $6,3 \%$ & 105 & $93,8 \%$ & \\
\hline & 2 & 2 & $3,3 \%$ & 58 & $96,7 \%$ & \\
\hline & 3 & 5 & $16,1 \%$ & 26 & $83,9 \%$ & \\
\hline & 4 & 0 & $0 \%$ & 18 & $100,0 \%$ & \\
\hline Edad & Prom & $3,9( \pm 1,3)$ & & $4,4( \pm 1,1)$ & & 0,084 \\
\hline Edad (madre) & Prom & $25( \pm 8)$ & & $24( \pm 6)$ & & 0,474 \\
\hline $\begin{array}{l}\text { T. inicio alimen. } \\
\text { Comp. }\end{array}$ & Prom & $1,2( \pm 1,3)$ & & $4,8( \pm 2,8)$ & & \\
\hline
\end{tabular}

Para tipo de familia, se encontró que la proporción de lactancia materna en padres separados fue de 81,5\%, Nuclear 95,1\%, Monoparental 87,5\%, Madre soltera 89,1\%, Familia Monoparental 100,0 \%, Consanguinidad extendida 100,0\%.

bronquiolitis $(15,8 \%$, IC 95\%:12\%-20,5\%), rinitis (13,9\%, IC 95\%:10,3\%-18,3\%), neumonía (10,6\%, IC 95\%:7,5\%-14,8\%), dermatitis atópica (9,7\%, IC 95\%:6,7\%-13,7\%), reflujo gastroesofágico (9,4\%, IC 95\%:6,3-13,3\%), asma (7,8\%, IC 95\%:5,1\%$11,5 \%)$, desnutrición $(5,8 \%, 3,6 \%-9,2 \%)$, afección cardiorrespiratoria (4,9\%, IC 95\%:2,9\%-8,1\%), dermatitis de contacto (3,7\%, IC 95\%:1,9\%-6,5\%), obesidad $(2,6 \%, 1,2 \%-5,2 \%)$, sinusitis $(2,9 \%$, IC 95\%:1,4\%-5,6\%), síndrome obstructivo (1,7\%, IC 95\%:0,6\%-4\%), diabetes (1,3\%, IC 95\%:0,4\%-3,5\%), apendicitis (0,6\%, IC 95\%:0,1\%-2,6\%), otras patologías (varicela, virosis, anemia, cólicos, otras, 15,2\%, IC 95\%:11,1\%-20,2\%). El 16,1\% de los participantes en el estudio (IC 95\%: 12,3\%-20,8\%) presentaban al momento de la encuesta alguna patología, dentro de las cuales se encontraron en orden de frecuencia: rinofaringitis (11,1\%), amigdalitis $(8,9 \%)$, herpes oral y virosis $(4,4 \%)$, alteración respiratoria $(2,2 \%)$ en orden de frecuencia.

\section{Relaciones entre variables}

Se encontró que existe una relación significativa entre lactancia materna (como factor protector) 
con bronquiolitis, síndrome bronco obstructivo, reflujo gastroesofágico, desnutrición, y patología actual, como se puede observar en la tabla 2. No se encontró relación significativa entre estas patologías y el tipo de familia.

Se encontró asociación significativa entre apendicitis $(p=0,031)$, neumonía $(p=0,007)$ y afección cardiorrespiratoria $(\mathrm{p}=0,000)$ con tiempo de lactancia. El promedio de tiempo de lactancia para los que presentaron apendicitis fue 8,5 meses y los que no, 10,78 meses. El tiempo de lactancia materna para los que padecieron neumonía fue de 7,73 meses mientras que para los que no padecieron la patología fue de 11,12 meses. Con respecto a la afección cardiorrespiratoria, el promedio de tiempo con lactancia materna para los que la tuvieron fue de 5,5 meses, en comparación con un promedio de 10,95 meses para aquellos que no la tuvieron. En cuanto al tipo de familia y tiempo de lactancia, no se encontró asociación estadísticamente significativa $(\mathrm{p}=0,762)$.

\section{DISCUSIÓN}

En el presente estudio se encontró la lactancia materna como factor protector para enfermedades de la infancia, especialmente bronquiolitis, síndrome bronco obstructivo, reflujo gastroesofágico, desnutrición, afección cardiorrespiratoria, y estado de enfermedad, en el momento de responder el instrumento. Se observaron hallazgos similares a los de varios estudios reportados en la literatura, entre ellos el estudio realizado por $\mathrm{Juez}^{8}$ y Colaboradores en Chile, en el hospital Paula Jaraquemada en una población de 242 niños; el estudio retrospectivo efectuado por Carvajal ${ }^{11}$ y colaboradores en México en el Policlínico Docente Comunitario "Victoria de Girón" en el 2003. O el realizado en el Reino Unido entre el 2000 y el 2002 por Quigley ${ }^{12}$ y colaboradores, donde los datos analizados demuestran que la lactancia materna exclusiva, en comparación con la no lactancia materna, protege contra la hospitalización e infecciones de las vías respiratorias. Además, el estudio realizado en 1998 por Calzado y col en el policlínico Armando García Aspurú, ${ }^{13}$ se encontró una relación entre la lactancia materna y la enfermedad respiratoria aguda. Oceguera y colaboradores, ${ }^{6}$ en un trabajo publicado en el año 2005 y realizado en una institución de salud de la Ciudad de México, encontró que la lactancia materna resultó ser factor protector para la presencia de infecciones respiratorias y gastrointestinales.

\section{Tabla 2. Patologías que mostraron relación con lactancia materna en estudiantes de 1 a 5 años de edad de diversas instituciones que participaron en el estudio.}

\begin{tabular}{|c|c|c|c|c|c|c|}
\hline \multirow{3}{*}{ Patología } & & \multicolumn{4}{|c|}{ Lactancia Materna } & \multirow{3}{*}{$\mathbf{P}$} \\
\hline & & \multicolumn{2}{|c|}{ Si } & \multicolumn{2}{|c|}{ No } & \\
\hline & & $\mathbf{N}$ & $\%$ & $\mathbf{N}$ & $\%$ & \\
\hline \multirow{2}{*}{ Bronquiolitis } & $\mathrm{Si}$ & 40 & 14 & 9 & 36 & \multirow{2}{*}{0,004} \\
\hline & No & 245 & 86 & 16 & 64 & \\
\hline \multirow{2}{*}{ Síndrome bronco obstructivo } & $\mathrm{Si}$ & 3 & 1,1 & 2 & 10,5 & \multirow[b]{2}{*}{0,002} \\
\hline & No & 265 & 98,9 & 17 & 89,5 & \\
\hline \multirow{2}{*}{ Reflujo gastroesofágico } & $\mathrm{Si}$ & 23 & 8,3 & 5 & 22,7 & \multirow{2}{*}{0,026} \\
\hline & No & 253 & 91,7 & 17 & 77,3 & \\
\hline \multirow{2}{*}{ Desnutrición } & $\mathrm{Si}$ & 14 & 4,9 & 4 & 16,0 & \multirow{2}{*}{0,023} \\
\hline & No & 270 & 95,1 & 21 & 84,0 & \\
\hline \multirow{2}{*}{ Patología actual } & $\mathrm{Si}$ & 41 & 14,7 & 8 & 32,0 & \multirow{2}{*}{0,029} \\
\hline & No & 238 & 85,3 & 17 & 68,0 & \\
\hline
\end{tabular}


Entre 1999 y 2002, Gómez-Alcalá y HurtadoGuzmán ${ }^{14}$ estudiaron en un Hospital de la Ciudad Obregón (México) la posibilidad de que la lactancia materna brinde protección contra el desarrollo de apendicitis en la vida futura. El riesgo de padecer apendicitis aumenta en los niños que no recibieron alimentación exclusiva del seno materno. Hay discusión sobre la falta de lactancia materna y la dermatitis, se argumenta que podría estar relacionado con el tipo de alimentación de la madre. ${ }^{15}$

Los resultados de este estudio en cuanto a lactancia materna como factor protector para enfermedad cardiorrespiratoria, coinciden con informes que señalan que la lactancia por más de seis meses protege a los niños de enfermedades cardiorrespiratorias en la vida adulta ya que disminuye el riesgo de un síndrome metabólico, pues se encuentra que en los niños que fueron lactados hay menor perímetro abdominal y menos obesidad. ${ }^{16}$ De otra parte, Goycochea-Valdivia ${ }^{17} \mathrm{y}$ colaboradores en su trabajo publicado en el año 2010, realizado en el Hospital Nacional Cayetano Heredia del Perú, muestran asociación entre lactancia materna y rinitis.

García y Acosta-Ramírez realizaron un estudio que se desarrolló en noviembre del 2003 a 1004 mujeres residentes en barrios de estratos socioeconómicos bajo, bajo-medio y medio, del área urbana de 4 ciudades colombianas: Cali, Cartagena, Medellín e Ibagué; allí se halló relación entre el tipo de familia y el tiempo de lactancia materna dado al niño, encontrando que las madres sin pareja lactaban durante 4,3 meses y las madres con pareja durante 4,4 meses. ${ }^{1}$

Dei-Cas y colaboradores ${ }^{18}$ mostraron en su trabajo, efectuado en 258 niños entre 2 y 9 años de edad, que el sobrepeso y la obesidad estaban relacionados con tiempos de lactancia menores de 3 meses.

En el presente estudio no se encontró asociación entre lactancia materna y enfermedad diarreica aguda como afirman varios autores. ${ }^{11,19,20}$
En cuanto a las limitaciones del estudio, se encuentra el tipo de muestreo que limita la generalización de los resultados así como que el instrumento haya sido autorespondido por las pacientes y los datos no hayan sido validados por otro método (historia clínica) lo que podría llevar a un riesgo de mala clasificación. Dada la metodología utilizada, no fue posible volver a acceder a cada madre para verificar los datos obtenidos en la encuesta inicial.

\section{AGRADECIMIENTOS}

Los autores agradecen al Doctor Eduardo Castaño Molina, M.G Rodrigo Rengifo Cárdenas, a la Doctora Margarita Loaiza y al señor Álvaro Restrepo Meza.

\section{REFERENCIAS}

1. Rodríguez-García J, Acosta-Ramírez N. Factores asociados a la lactancia materna exclusiva en población pobre de áreas urbanas de Colombia. Rev Salud Pública 2008;10:SP PUJ 2008;10:71-84.

2. Díaz-Tabare O, Soler-Quintana L, Ramos-Rodríguez AO, González-Mason L. Aspectos epidemiológicos relacionados con el tipo de lactancia durante el primer año de vida. Rev Cubana Med Gen integr 2001;17:336-43.

3. Segura-Rosero AM, Maestre C, Cure-Cure C, LatorrePadavi H. Influencia de lactancia materna exclusiva o alimentación temprana con fórmula sobre las enfermedades durante la niñez. Revista de Alergia, Asma e Inmunología 2002;11:66-72.

4. Eckhart CL, Rivera J, Adaid IS, Martorell R. Full breast-feeding for at least four month has differential effects on growth before and after six months of age among children in Mexican community. J Nutr 2001;131:2304-9.

5. Díaz-Gómez NM, Doménech E. Avances en lactancia materna. Can Ped 2000;24:39-46.

6. Oceguera-Hernández VC, Gutiérrez-Muñoz J, LunaRuiz J, Alva-Valdez J. Lactancia materna, infecciones gastrointestinales y respiratorias. Rev Esp Med Quir 2005;10:30-4.

7. González-Hoyos DM. La lactancia materna como ambiente facilitador del desarrollo de niño. Manizales: Universidad de Caldas, Colciencias; 2003. 
8. Juez G, Díaz S, Peralta O, Croxatto HB, Casado ME, Salyattierra AM, et al. Lactancia materna exclusiva: crecimiento del lactante en un grupo seleccionado de niños Chilenos. Rev Chil Pediatr 1981;55:225-30.

9. Manrique M, Corredor Martínez N, Nieto B. Por una niñez bien nutrida: comunicación para la acción. 3a. ed. Bogotá: Editorial Fondo de las Naciones Unidas para la Infancia. (UNICEF); 2004.

10. Carratalá-Munueraa MC, Gascón-Pérez E, RagaOrtega M. ¿Es la lactancia materna un factor de protección ante los procesos infecciosos? Estudio de casos y controles. Aten Primaria 2005;35:140-5.

11. Coronel-Carvajal C, Hernández-Cisneros F, MartínArgilagos M. Lactancia materna en el primer semestre y la salud de los niños en el primer año de vida. Rev Mex Pediatr 2004;71:217-21.

12. Quigley MA, Yvonne J, Sacker K, Sacker A. Breastfeeding and Hospitalization for Diarrheal and Respiratory Infection in theUnited Kingdom Millennium, Cohort Study. Pediatrics 2007;1 19:837-42.

13. Calzado-Mustelier M, Rodríguez-Rivero L, VargasFajardo E, Vistel-Sánchez M. Influencia de la lactancia materna en la salud del niño. Rev Cubana Enfermer 2000;16:122-7.

14. Gómez-Alcalá AV, Hurtado-Guzmán A. El destete precoz como factor de riesgo en apendicitis aguda en niños. Gac Méd Méx 2005;141:501-4.
15. Magaña-García M, Reyes-Vázquez H. Influencia de la lactancia materna en el desarrollo de la dermatitis atópica en una pareja de gemelas. Rev Med Hosp Gen Mex 1999;62:202-5.

16. Rudnicka AR, Owen CG, Strachan DP. The effect of breastfeeding on cardiorespiratory risk factors in adult life. Pediatrics 2007;119:1107-15.

17. Goycochea-Valdivia WA, Hidalgo-Tunque CM, Hernández-Díaz H, Centeno-Huaman J. Asociación entre prematuridad, bajo peso al nacer y lactancia materna exclusiva con rinitis alérgica, en niños de 2 a 7 años que acuden al Hospital Nacional Cayetano Heredia, Perú. Bol Med Hosp Infant Mex 2010;67:315-26.

18. Dei-Cas PG, Dei-Cas SA, Dei-Cas IJ. Sobrepeso y obesidad en la niñez. Relación con factores de riesgo. Arch argent pediatr 2002;100:368-73.

19. Macías-Carrillo C, Franco-Marina F, Long-Dunlap K, Hernández-Gaytán SI, Martínez-López Y, LópezCervantes M. Lactancia materna y diarrea aguda en los primeros tres meses de vida. Salud Pública Méx 2005;47:49-57.

20. Salazar S, Chávez M, Delgado X, Pacheco T, Rubio E. Lactancia materna. Arch Venezol Puericult 2009;72:163-6. 